\title{
Cramér-Rao Lower Bounds for the Time Delay Estimation of UWB Signals
}

\author{
J. Zhang, R. A. Kennedy, T. D. Abhayapala \\ Department of Telecommunications Engineering, RSISE \\ The Australian National University, Canberra ACT 0200, Australia \\ Email: jian@syseng.anu.edu.au
}

\begin{abstract}
In this paper, we present the Cramér-Rao lower bounds (CRLBs) for the time delay estimation of UWB signals which could be tight lower bounds for the theoretical performance limits of UWB synchronizers. The CRLBs are investigated for both single pulse systems and time hopping systems in AWGN and multipath channels. Insights are given into the relationship between CRLBs for different Gaussian monocycles. It is found that larger number of multipath signals implies higher CRLBs and inferior performance of synchronizers, and multipath interference on CRLBs can not be eliminated completely except in very special cases. As every estimate of time delay could not be perfect, the least influence of the synchronization error on the performance of receivers is quantified.
\end{abstract}

\section{INTRODUCTION}

Ultra Wideband (UWB) is a promising technique in the application of short-range high-speed wireless communication and precise location tracking. Typically, ultra narrow pulses, such as Gaussian monocycles [1], are modulated to transmit information. These pulses could be narrower than 1 nanosecond. This brings very stringent synchronization requirements.

A UWB signal is basically a baseband signal without phase and carrier information, hence time delay estimation is the main task of a synchronizer. This synchronizer could be one in a simple single-pulse UWB system, however, due to the power limitation imposed by FCC [2], UWB pulses are generally combined with spread spectrum techniques, especially time hopping (TH). Like in traditional spread spectrum systems, the synchronization of a time-hopping UWB system can be accomplished in two steps: code acquisition followed by code tracking.

Some research on the design and performance of UWB synchronizers has been reported in [3]-[6]. In order to provide benchmarks for these synchronizers, it is important to understand the theoretical performance limits of synchronizers. Among these limits, the Cramér-Rao Lower Bound (CRLB) is most widely used. The CRLB [7] is a fundamental lower bound on the variance of any unbiased estimator. The analysis of CRLB for synchronizers in traditional systems is well founded (e.g., see [8]-[11]), but for UWB, there is no systematic work yet. This paper is concerned with evaluating the CRLB of UWB synchronizers for different UWB pulses. Both single-pulse systems and time-hopping systems are considered. For time hopping, the CRLB can be a lower bound for the performance of code tracking. The evaluation of CRLBs is generally mathematically quite difficult when the observed signal contains, besides the parameter to be estimated, also some nuisance parameters that are unknown [9], [11]. To generate results intuitively, we only consider unmodulated UWB signals here.

This paper is organized as follows. Section II introduces the system model. In Section III, the CRLB for single-pulse systems in AWGN channels is discussed and some insights into the relationship between CRLBs for different Gaussian monocycles are given. Section IV derives the CRLBs for UWB signals in a multipath channel. The influence of synchronization error on the performance of receiver is quantified in a simple example in Section V. Finally, numerical results are given in Section VI to verify the analytical results.

\section{SYSTEM STRUCTURE}

Let $s(t)$ be the transmitted UWB signal. In an unmodulated single-pulse system, $s(t)=\sum_{i} \omega\left(t-i T_{s}\right)$, where $\omega(t)$ is a UWB pulse, $T_{s}$ is the symbol period. In an unmodulated time hopping system, $s(t)=\sum_{i} s_{i}(t)=\sum_{i} \sum_{j=1}^{N_{f}} \omega\left(t-i T_{s}-\right.$ $\left.j T_{f}-c_{j} T_{c}\right)$ where $T_{f}$ is the frame width, $N_{f}$ is the number of frames in a symbol, $T_{c}$ is the chip width, and $c_{j}$ is the time hopping code.

The UWB pulses considered are series of Gaussian monocycles $\omega\left(t ; n, t_{p}\right)$, which are scaled and/or differentiated versions of the basic Gaussian waveform $\omega_{0}(t)=\exp \left(-2 \pi t^{2}\right)$, that is, $\omega\left(t ; n, t_{p}\right)=\omega_{0}^{(n)}\left(t / t_{p}\right)$, where the superscript ${ }^{(n)}$ stands for $n$-order differentiation with respect to $t$.

To ensure equal energy of monocycles, a coefficient $\varepsilon\left(n, t_{p}\right)$ is introduced, and let $\omega(t)=\varepsilon\left(n, t_{p}\right) \omega\left(t ; n, t_{p}\right)$. Denote the energy of $\omega(t)$ as $E_{p}$, then $\varepsilon\left(n, t_{p}\right)$, depending on $n$ and $t_{p}$, satisfies

$$
\varepsilon^{2}\left(n, t_{p}\right)=\frac{E_{p}}{\int_{-\infty}^{+\infty} \omega^{2}\left(t ; n, t_{p}\right) d t}
$$

In a pure AWGN channel $n(t)$, the received signal $r(t)$ is

$$
r(t)=s(t-\tau)+n(t)
$$

where every sample of $n(t)$ is Gaussian distributed with zero mean and variance $\sigma_{0}^{2}$, and $\tau$ is the timing delay to be estimated. 
In a selective fading channel, $h(t)=\sum_{\ell=1}^{L} a_{\ell} \delta\left(t-\tau_{\ell}\right)$, the received signal is given by

$$
r(t)=\sum_{\ell=1}^{L} a_{\ell} s\left(t-\tau_{\ell}\right)+n(t),
$$

where $a_{\ell}$ and $\tau_{\ell}$ are real multipath gains and delays, respectively. Note the time delay $\tau$ between transmitter and receiver is merged into $\tau_{\ell}$.

Due to the low duty cycle of UWB signals, we assume the received signal is free of intersymbol interference (ISI) unless indicated otherwise.

For the AWGN model in (2), estimated based on $K$ independent observations, the received signal can be represented as a vector model

$$
\mathbf{r}=\mathbf{s}+\mathbf{n}
$$

where $\mathbf{r}=\left[r_{1}, \cdots, r_{K}\right], \mathbf{s}=\left[s_{1}, \cdots, s_{K}\right]$ and $\mathbf{n}=$ $\left[n_{1}, \cdots, n_{K}\right]$.

Suppose an unbiased estimate $\hat{\tau}$ of the time delay $\tau$ can be generated from (4), then the estimation error variance is lower bounded by the CRLB $E_{\mathbf{r}}\left[(\hat{\tau}-\tau)^{2}\right] \geq \operatorname{CRLB}(\tau)$, where

$$
\operatorname{CRLB}(\tau)=\left(E_{\mathbf{r} \mid \tau}\left[-\frac{d^{2}}{d \tau^{2}} \ln (p(\mathbf{r} \mid \tau))\right]\right)^{-1}
$$

In (5), the conditional pdf $p(\mathbf{r} \mid \tau)$ is the likelihood function of $\tau$, and the expectation $E_{\mathbf{r} \mid \tau}[\cdot]$ is with respect to $p(\mathbf{r} \mid \tau)$.

Since the additive noise $n(t)$ is white and zero mean, $p(\mathbf{r} \mid \tau)$ can be expressed as

$$
\begin{aligned}
p(\mathbf{r} \mid \tau) & =\prod_{k=1}^{K} \frac{1}{\sqrt{2 \pi} \sigma_{0}} \exp \left(-\frac{1}{2 \sigma_{0}^{2}}\left(r_{k}-s_{k}\right)^{2}\right) \\
& =\left(\frac{1}{\sqrt{2 \pi} \sigma_{0}}\right)^{K} \exp \left(-\frac{1}{2 \sigma_{0}^{2}} \sum_{k=1}^{K}\left(r_{k}-s_{k}\right)^{2}\right) .
\end{aligned}
$$

A continuous-time equivalent of $p(\mathbf{r} \mid \tau)$ can be developed [7, p.274] [12, p.335], and the log-likelihood function $\mathcal{L}(\mathbf{r} ; \tau)$ has the form

$$
\mathcal{L}(\mathbf{r} ; \tau)=\frac{1}{2 \sigma_{0}^{2}}\left(2 \int_{T_{o}} r(t) s(t-\tau) d t-\int_{T_{o}} s^{2}(t-\tau) d t\right)
$$

The process from (4) to (7) can be applied to the multipath model (3) with minor modifications.

\section{CRLB FOR SINGLE-PULSE SYSTEMS IN AWGN CHANNELS}

In this case, the CRLB, further derived from (7) or directly from [13], has the form

$$
\operatorname{CRLB}(\tau)=\frac{\sigma_{0}^{2}}{\int_{T_{o}} \dot{s}^{2}(t-\tau) d t},
$$

where $\dot{s}(t-\tau)$ denotes once partial differentiation with respect to $\tau$.

Assuming that the pulse is strictly restricted within a symbol period, the denominator in (8) equals $N \int_{T_{s}} \dot{\omega}^{2}(t-\tau) d t$, where $N=T_{o} / T_{s}$ is the number of symbols in the observation period. For a specific monocycle, the lower variance bound becomes

$$
\operatorname{CRLB}(\tau)=\frac{1}{N \gamma_{s}} \frac{\int_{T_{s}} \omega^{2}\left(t-\tau ; n, t_{p}\right) d t}{\int_{T_{s}} \dot{\omega}^{2}\left(t-\tau ; n, t_{p}\right) d t},
$$

where the symbol SNR $\gamma_{s}=E_{p} / \sigma_{0}^{2}$.

If the symbol period $T_{s}$ is large enough so that most of the energy of the pulse concentrates within $T_{s}$, we can express (9) in frequency domain

$$
\operatorname{CRLB}(\tau)=\frac{1}{N \gamma_{s}} \frac{\int_{-\infty}^{+\infty}\left|W\left(f ; n, t_{p}\right)\right|^{2} d f}{\int_{-\infty}^{+\infty} f^{2}\left|W\left(f ; n, t_{p}\right)\right|^{2} d f},
$$

where $W\left(f ; n, t_{p}\right)$ is the Fourier Transform of $\omega\left(t ; n, t_{p}\right)$.

According to the properties of the Fourier Transform of derivatives of functions, we find explicit relationships exist between the CRLBs of monocycles with different $n$ but same $t_{p}$, that is,

$$
\begin{aligned}
& \frac{\operatorname{CRLB}(\tau)_{n}}{\operatorname{CRLB}(\tau)_{n+1}} \\
& =\frac{\int_{-\infty}^{+\infty}\left|W\left(f ; n, t_{p}\right)\right|^{2} d f \cdot \int_{-\infty}^{+\infty} f^{4}\left|W\left(f ; n, t_{p}\right)\right|^{2} d f}{\left(\int_{-\infty}^{+\infty} f^{2}\left|W\left(f ; n, t_{p}\right)\right|^{2} d f\right)^{2}} \\
& >1,
\end{aligned}
$$

where the inequality is an application of Schwarz's inequality. This inequality implies that monocycles with higher order differentiation have the potential for better performance in the sense of lower synchronization error variance.

For monocycles with different $t_{p}$ but same $n$, the ratio between their CRLBs can be found as

$$
\frac{\operatorname{CRLB}(\tau)_{t_{p 1}}}{\operatorname{CRLB}(\tau)_{t_{p 2}}}=\left(\frac{t_{p 1}}{t_{p 2}}\right)^{2},
$$

which implies that monocycles with smaller $t_{p}$ (narrower effective pulse width) have the potential for better synchronization performance.

\section{CRLB FOR TIME-HOPPING UWB SYSTEMS IN SELECTIVE-FADING CHANNELS}

When the channel is AWGN, the analysis and results in Section III can be applied to time hopping UWB systems with minor modification. The change can be merged into the symbol SNR $\gamma_{s}$, that is, $\gamma_{s}$ equals to the ratio between the energy of $N_{f}$ pulses and the noise variance $\sigma_{0}^{2}$ for TH UWB systems. In this section, we will focus on selective fading channels and derive the CRLBs using joint detection for multiple multipath parameters $\mathbf{a}=\left[a_{1}, \ldots, a_{\ell}, \ldots, a_{L}\right]_{1 \times L}$ and $\tau=\left[\tau_{1}, \ldots, \tau_{\ell}, \ldots, \tau_{L}\right]_{1 \times L}$, which are treated as unknown but deterministic.

Start with (3), the log-likelihood function in (7) can be rewritten as $\mathcal{L}(\mathbf{r} ; \tau, \mathbf{a})$ as

$$
\begin{aligned}
\mathcal{L}(\mathbf{r} ; \tau, \mathbf{a})= & \frac{1}{\sigma_{0}^{2}} \int_{T_{o}} r(t) \sum_{\ell} a_{\ell} s\left(t-\tau_{\ell}\right) d t- \\
& \frac{1}{2 \sigma_{0}^{2}} \int_{T_{o}}\left[\sum_{\ell} a_{\ell} s\left(t-\tau_{\ell}\right)\right]^{2} d t
\end{aligned}
$$


Lower bounds on the variances of estimates for the components of $a_{\ell}$ and $\tau_{\ell}$ are given in terms of the diagonal elements of the inverse of the Fisher information matrix $\mathbf{J}^{-1}$ [7]. After some manipulation, the Fisher Information Matrix $\mathbf{J}$ can be written as

$$
\mathbf{J}=\left(\begin{array}{ll}
J_{\tau \tau} & J_{\tau \mathbf{a}} \\
J_{\mathbf{a} \tau} & J_{\mathbf{a a}}
\end{array}\right)
$$

where $J_{\tau \tau}, J_{\tau \mathbf{a}}, J_{\mathbf{a} \tau}$ and $J_{\text {aa }}$ are all $L \times L$ matrices with $[\ell, m]^{t h}$ elements

$$
\begin{aligned}
& J_{\tau \tau}[\ell, m]=\frac{1}{\sigma_{0}^{2}} \int_{T_{o}} a_{\ell} a_{m} \dot{s}\left(t-\tau_{\ell}\right) \dot{s}\left(t-\tau_{m}\right) d t \\
& J_{\mathbf{a a}}[\ell, m]=\frac{1}{\sigma_{0}^{2}} \int_{T_{o}} s\left(t-\tau_{\ell}\right) s\left(t-\tau_{m}\right) d t \\
& J_{\tau \mathbf{a}}[\ell, m]=J_{\mathbf{a} \tau}[m, \ell]=-\frac{1}{\sigma_{0}^{2}} \int_{T_{o}} a_{\ell} \dot{s}\left(t-\tau_{\ell}\right) s\left(t-\tau_{m}\right) d t
\end{aligned}
$$

respectively.

The CRLB for $\tau_{\ell}$ is just the $\ell^{t h}$ diagonal element of the inverse of $\mathbf{J}$. Use $\ell=1$ as an example and rewrite the matrix $\mathbf{J}$ as

$$
\mathbf{J}=\left(\begin{array}{cc}
J_{11} & \mathbf{B} \\
\mathbf{C} & \mathbf{D}
\end{array}\right)
$$

we have

$$
\begin{aligned}
\operatorname{CRLB}\left(\tau_{1}\right) & =J_{11}^{-1}+J_{11}^{-1} \mathbf{B}\left(\mathbf{D}-\mathbf{C} J_{11}^{-1} \mathbf{B}\right)^{-1} \mathbf{C} J_{11}^{-1} \\
& =J_{11}^{-1}+J_{11}^{-2} \mathbf{B}{\widetilde{J_{11}}}^{-1} \mathbf{C} \\
& \geq J_{11}^{-1}
\end{aligned}
$$

where $\widetilde{J_{11}}$ is called the Schur complement of $J_{11}[14, \mathrm{p} .175]$. Since $\mathbf{J}$ is nonnegative definite, the Schur complement matrix $\widetilde{J_{11}}$ is also nonnegative definite, so is ${\widetilde{J_{11}}}^{-1}$. At the same time, $\mathbf{B}$ is the transpose of $\mathbf{C}$ since $\mathbf{J}$ is a symmetric matrix in this case. Thus we get $\mathbf{B} \widetilde{J_{11}} \mathbf{C} \geq 0$ and the inequality in (22) follows immediately. When utilize the knowledge of $J_{11}>$ 0 according to (16), we can get the inequality in (22) more readily according to

$$
\operatorname{CRLB}\left(\tau_{1}\right)=\left(J_{11}-\mathbf{B D}^{-1} \mathbf{C}\right)^{-1}>J_{11}^{-1} .
$$

As $J_{11}^{-1}$ can be regarded as the CRLB in an AWGN channel with a known scalar of amplitude, this inequality implies the CRLB in joint detection is always larger than that in the single parameter estimation in an AWGN channel. Then an interesting question arises, whether more multipath means higher CRLB and inferior performance of synchronizer accordingly?

Let us consider a channel with $L-1$ multipath signals. The Fisher Information Matrix $\mathbf{J}^{\prime}$ can be written as

$$
\mathbf{J}^{\prime}=\left(\begin{array}{cc}
J_{11} & \mathbf{B} \\
\mathbf{C} & \mathbf{D}^{\prime}
\end{array}\right),
$$

with

$$
\mathbf{D}^{\prime}=\left(\begin{array}{cc}
\mathbf{D}_{1} & \mathbf{0} \\
\mathbf{0}^{\dagger} & 0
\end{array}\right)
$$

where $\mathbf{0}$ is a $(L-2) \times 1$ zero vector and ${ }^{\dagger}$ stands for transpose operation. Then the CRLB with $L-1$ multipath is

$$
\operatorname{CRLB}\left(\tau_{1}\right)_{L-1}=\left(J_{11}-\mathbf{B D}^{\prime-1} \mathbf{C}\right)^{-1} .
$$

Comparing $\mathbf{B D}^{-1} \mathbf{C}$ and $\mathbf{B D}^{\prime-1} \mathbf{C}$ gives

$$
\begin{aligned}
\mathbf{B D}^{-1} \mathbf{C}-\mathbf{B D}^{\prime-1} \mathbf{C} & =\mathbf{B}\left(\mathbf{D}^{-1}-\left(\begin{array}{cc}
\mathbf{D}_{1}^{-1} & \mathbf{0} \\
\mathbf{0}^{\dagger} & 0
\end{array}\right)\right) \mathbf{C} \\
& \geq 0,
\end{aligned}
$$

where the inequality in (28) yields from that $\mathbf{D}^{-1}-\mathbf{D}^{\prime-1}$ is a nonnegative definite matrix as can be proven according to the property of partitioned nonnegative definite matrices (e.g., see [14, p178], let $\mathbf{D}^{-1}=\mathbf{A}$ in equation (6.10)).

Recall $J_{11}>0$, we have

$$
\operatorname{CRLB}\left(\tau_{1}\right)_{L}>\operatorname{CRLB}\left(\tau_{1}\right)_{L-1},
$$

which shows that more multipath does lead to higher CRLB and inferior performance of synchronizer. Since the number of multipath is closely relevant to the bandwidth of monocycles, we conclude that narrower monocycles will very likely cause larger CRLBs. We did not say "absolutely" because all other variables besides D during this derivation are assumed unchanged, but it could be unrealistic when different monocycles are applied.

Another key factor with influence on CRLB is the choice of TH codes. When the autocorrelation of TH codes is ideal, the CRLBs in a multipath channel will be similar to the one in an AWGN channel.

So far, we have seen that the performance of synchronizers is deteriorated by the multipath interference. It is natural to ask whether the multipath interference can be mitigated or fully eliminated before entering the decision part of a synchronizer?

As shown for CDMA systems in [15], it is possible to remove part of multipath interference in UWB systems. However, unless the correlation of $\mathrm{TH}$ codes is ideal, the total removal of multipath interference is impossible due to the existence of $n(t)$. This is because, any estimate of parameters, including amplitude and delay, even though unbiased, may still have a nonzero variance in the present of noise. The CRLB can generally be achieved by Maximum Likelihood estimation asymptotically (when the number of observation samples goes to infinity), and the estimation error becomes Gaussian distributed with zero mean and variance equivalent to the CRLB [7], [8]. Therefore, the final signal with a pair of synchronization parameters of interest contains the sum of $2(L-1)$ Gaussian variables, which has a variance larger than the variance of $n(t)$. Since CRLB is proportional to the variance of (interference and) noise, the CRLBs for this pair of parameters will be larger than those in a single path channel. So no matter how perfect the structure and algorithm to remove multipath signal are, the effect of multipath interference can only be mitigated but can not be cancelled completely. This result also partly explains why more multipath generally leads to higher CRLBs. 
However, there are some special cases when multipath interference becomes negligible. For example, when the maximal multipath delay is smaller than the frame period in a single pulse system, multipath signals do not interfere with each other due to the low duty cycle of UWB signal structure.

\section{INFLUENCE OF SYNCHRONIZATION ERROR ON BER}

We discuss a simple example here to show the influence of synchronization error on the performance of receivers in UWB systems.

We consider a BPSK modulated single-pulse signal in an AWGN channel. A correlator receiver [16], [17] is used to detect the signal.

The conditional bit-error-ratio (BER), depending on the synchronization error $e_{\tau}$, is given by

$$
P_{e}\left(e_{\tau}\right)=Q\left(\frac{\rho\left(e_{\tau}\right)}{\sqrt{E_{p}} \sigma_{0}}\right),
$$

where $Q(x) \triangleq \int_{x}^{+\infty} \exp \left(-t^{2} / 2\right) / \sqrt{2 \pi} d t$ and $\rho\left(e_{\tau}\right)=$ $\int_{T_{s}} \omega(t) \omega\left(t-e_{\tau}\right) d t$.

Recall that the best achievable $e_{\tau}$ is Gaussian distributed with zero mean and variance equivalent to the CRLB (denoted by $\left.\sigma_{c}^{2}\right)$. In the best case, $\sigma_{c}^{2}=\sigma_{0}^{2} /\left(N \int_{T_{s}} \dot{\omega}^{2}(t-\tau) d t\right)$ from (8) is the smallest. Averaging $P_{e}\left(e_{\tau}\right)$ over $e_{\tau}$, we get the mean BER

$$
\begin{aligned}
P_{e} & =E\left[P_{e}\left(e_{\tau}\right)\right] \\
& =\int_{-\infty}^{+\infty} \frac{1}{\sqrt{2 \pi} \sigma_{c}} Q\left(\sqrt{\frac{\rho^{2}\left(e_{\tau}\right)}{E_{p} \sigma_{0}^{2}}}\right) \exp \left(\frac{-e_{\tau}^{2}}{2 \sigma_{c}^{2}}\right) d e_{\tau} .
\end{aligned}
$$

Statistically, this is the best achievable performance under certain SNR. This equation can be evaluated numerically by Monte Carlo simulation which requires highly computational complexity. Alternatively, we invoke the Hermite-Gauss quadrature [18], and $P_{e}$ can be accurately approximated by

$$
P_{e} \simeq \frac{1}{\sqrt{\pi}} \sum_{n=1}^{N_{h}} H_{x_{n}} Q\left(\frac{\rho\left(\sqrt{2} \sigma_{c} x_{n}\right)}{\sqrt{E_{p}} \sigma_{0}}\right),
$$

where $N_{h}$ is the order of the Hermite polynomial $H_{N_{h}}(\cdot)$, $x_{n}$ and $H_{x_{n}}$ are the zeros (abscissas) and weight factors of $N_{h}$-order Hermite polynomial, respectively. These values are tabulated in many mathematical handbooks (e.g., [19]). In experiments, we find first 16 coefficients $\left(N_{h}=16\right)$ are enough to generate accurate approximation results.

Further define a variable $\eta$ as the degrading ratio between $P_{e}$ and $P_{e}(0)=Q\left(\sqrt{\gamma_{s}}\right)$, which is the BER in the case of perfect synchronization. We show the values of $\eta$ for different monocycles in Section VI to compare the synchronization error robustness of monocycles.

\section{NUMERICAL RESULTS}

Since there is not widely acceptable UWB fading channel models yet, we only show numerical results on the CRLBs in pure AWGN channels.

In Fig. 1 - Fig. 3, the CRLBs for different monocycles are demonstrated. Since in practice, a transmitted monocycle is usually the truncated portion of a whole pulse $w\left(t ; n, t_{p}\right)$, this effect of truncation is considered by varying the actual width of pulse in (9).

From Fig. 1, we can see CRLBs are inversely proportional to symbol SNR and the observation period $N T_{s}$. The relationship between CRLBs for monocycles with different order $n$ coincides with the analytical results in (12). This can be further observed in Fig. 2, which also depicts the effect of truncated pulses on CRLB. The CRLBs change little even when the truncated portion narrows to $1.6 t_{p}$ (symmetric with respect to $t=0$ ). However, with the width of truncated pulse decreasing further, the CRLBs become orderless. Fig. 3 shows the effect of $t_{p}$ on the CRLBs, which is a direct verification of (13).
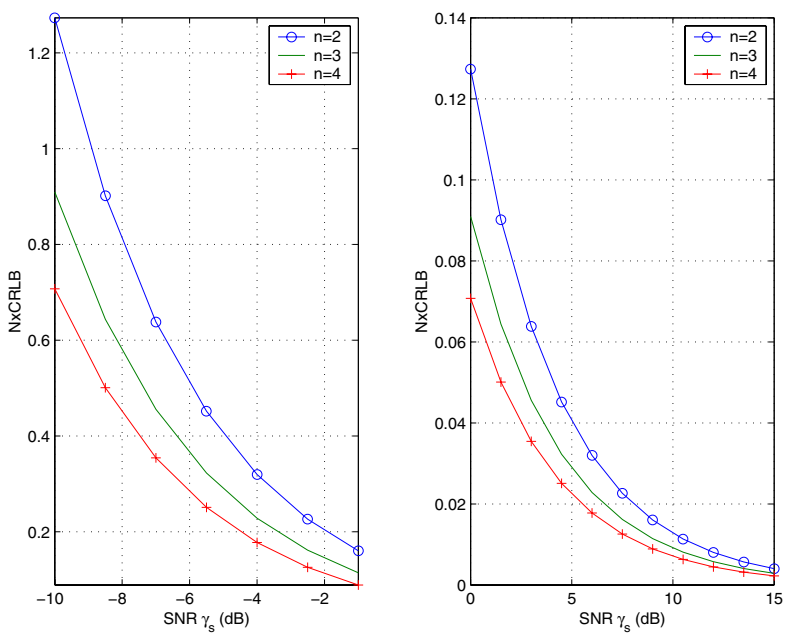

Fig. 1. CRLB versus symbol SNR $\gamma_{s}$ for $n$-order monocycles with $t_{p}=2$ ns, $n=2,3,4$.

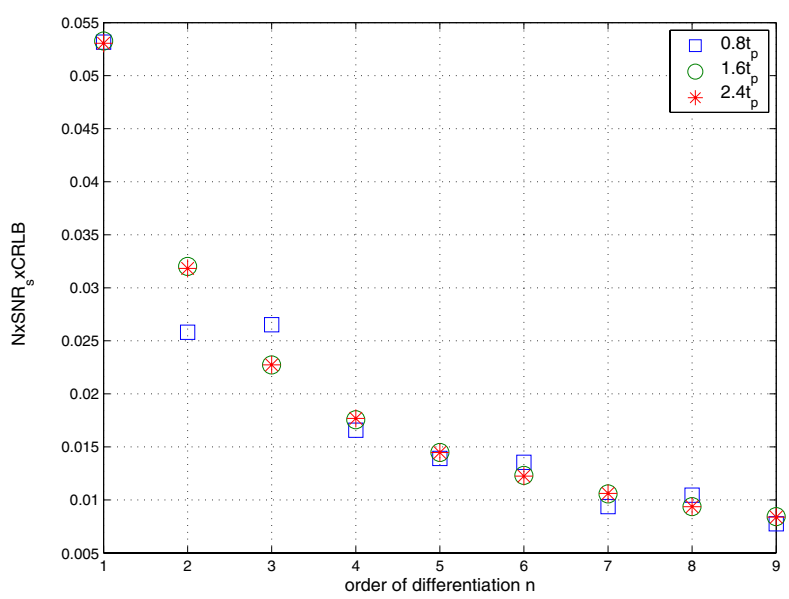

Fig. 2. CRLB versus order $n$ for monocycles with $t_{p}=1 \mathrm{~ns}$; different lines correspond to different width of truncation.

Fig. 4 demonstrates the influence of synchronization error on the performance of receivers. It is plotted from (32) using Hermite Gaussian approximation. The influence is notable when the observation window in the stage of synchronization has small width $\left(N T_{s}\right)$, and weakens with $N$ increasing 


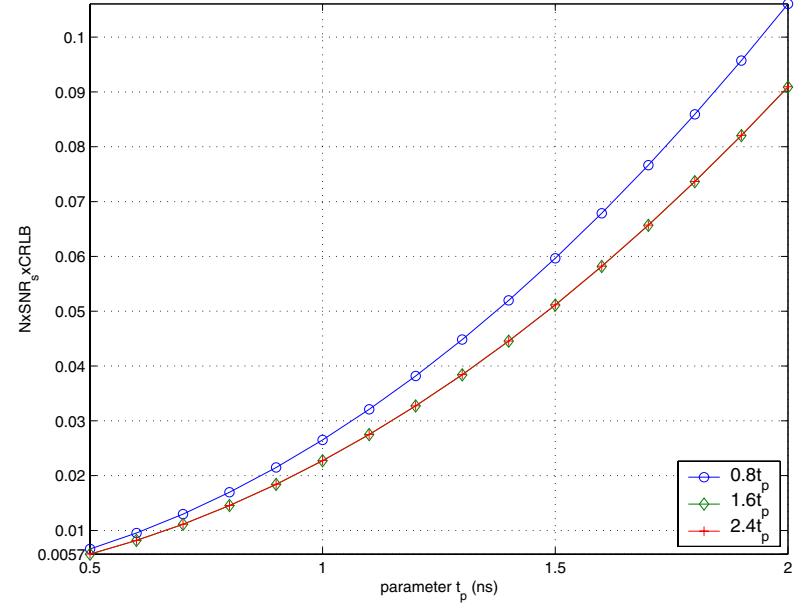

Fig. 3. CRLB for a 3 order $(n=3)$ monocycle with different parameter $t_{p}$; different lines correspond to different width of truncation.

(CRLBs decreasing). The figure also indicates that synchronization errors of different monocycles have very close influence on BER, although the data in experiments shows the influence of monocycles with larger $n$ is a little worse when SNR $\gamma_{s}$ is small, and changes toward opposite with SNR increasing.
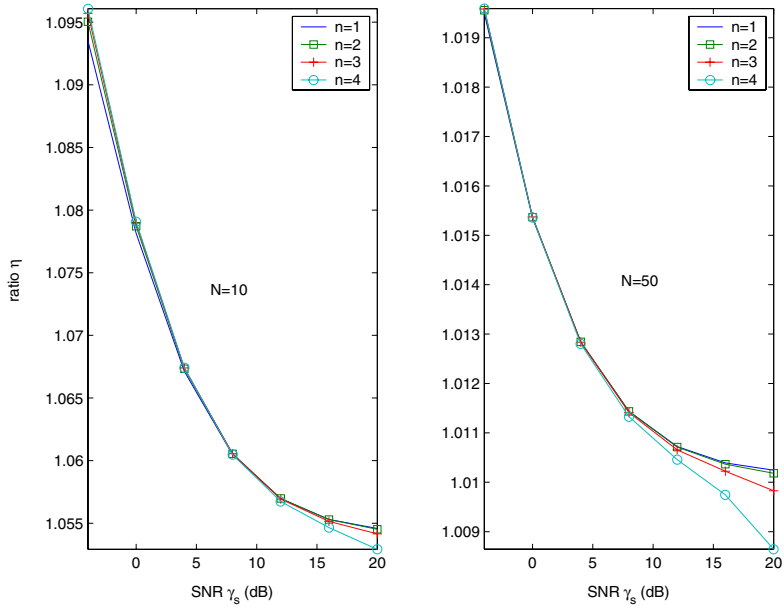

Fig. 4. The degrading ratio $\eta$ versus SNR $\gamma_{s}$ for monocycles. Two observation periods $\left(N T_{s}\right)$ in a synchronizer are compared with $N=10$ (left) and $N=50$ (right). The time $t$ is normalized with respect to $t_{p}$.

\section{CONCLUSIONS}

We have derived the Cramér-Rao lower bounds (CRLBs) for the time delay estimation of UWB signals for both single pulse systems and time hopping systems in AWGN and multipath channels. Insights are given on the relationship between CRLBs for different Gaussian monocycles. It is found that larger number of multipath implies higher CRLBs and inferior performance of synchronizers, and multipath interference on CRLBs can not be eliminated thoroughly except for in very limited cases. The influence of synchronization error on the performance of receivers is quantified in a simple example. The influence is notable when observation window $\left(N T_{s}\right)$ in a synchronizer is small, and weakens with $N$ increasing (CRLBs decreasing). Synchronization errors of different monocycles have very close influence on BER.

\section{REFERENCES}

[1] R. A. Scholtz, "Multiple access with time-hopping impulse modulation," in Proc. Military Communications Conf., Boston, MA, Oct. 1993, vol. 2, pp. 447-450.

[2] Federal Communication Committee, FCC: First Report and Order, Federal Communications Commission, U.S., April 22, 2002.

[3] E. A. Homier and R. A. Scholtz, "Rapid acquisition of Ultra-Wideband signals in the dense multipath channel," in Proc. IEEE Conf. on UWB Systems and Technologies (UWBST), May 2002.

[4] N. Rinaldi et al., "U.C.A.N.'s ultra wide band system: Baseband algorithm design," in Proc. International Workshop on UWB Systems, June 2003.

[5] I. Maravic and M. Vetterli, "Low-complexity subspace methods for channel estimation and synchronization in Ultra-Wideband systems," in Proc. International Workshop on UWB Systems, Oulu, Finland, June 2003.

[6] S. Soderi, J. Iinatti, and M. Hamalainen, "CLPDI algorithm in UWB synchronization," in Proc. International Workshop on UWB Systems, June 2003.

[7] Harry L. Van Trees, Detection, Estimation, and Modulation Theory, John Wiley \& Sons, Inc., New York, 1968.

[8] H. Meyr, M. Moeneclaey, and S. A. Fechtel, Digital Communication Receivers: Synchronization, Channel Estimation, And Signal Processing, A Wiley-Interscience Publication, John Wiley \& sons, Inc, New York, 1998.

[9] M. Moeneclaey, "On the true and the modified Cramer-Rao bounds for the estimation of a scalar parameter in the presence of nuisance parameters," IEEE Trans. Commun., vol. 46(11), pp. 1536-1544, Nov. 1998.

[10] E. G. Ström and F. Malmsten, "A maximum likelihood approach for estimating DS-CDMA multipath fading channels," IEEE J. Select. Areas Commun., vol. 18(1), pp. 132-140, Jan. 2000.

[11] F. Rice, B. Cowley, B. Moran, and M. Rice, "Cramer-Rao lower bounds for QAM phase and frequency estimation," IEEE Trans. Commun., vol. 49(9), pp. 1582-1591, Sep. 2001.

[12] J. G. Proakis, Digital Communications, McGraw-Hill, third edition, 1995.

[13] A. N. D’Andrea, U. Mengali, and R. Reggiannini, "The modified Cramer-Rao bound and its application to synchronization problems," IEEE Trans. Commun., vol. 42(2/3/4), pp. 1391-1399, Feb./Mar./April 1994.

[14] F. Zhang, Matrix Theory: Basic Results and Techniques, Springer-Verlag New York Inc., New York, 1999.

[15] G. fock, J. Baltersee, P. Schulz-Rittich, and H. Meyr, "Channel tracking for rake receivers in closely spaced multipath environments," IEEE J. Select. Areas Commun., vol. 19, pp. 2420-2431, Dec. 01.

[16] M. Z. Win and R. A. Scholtz, "Ultra-wide bandwidth time-hopping spread-spectrum impulse radio for wireless multiple-access communications," IEEE Trans. Commun., vol. 48(4), pp. 679-691, Apr. 2000.

[17] J. Zhang, T. D. Abhayapala, and R. A. Kennedy, "Performance of Ultra Wideband correlator receiver using gaussian monocycles," in Proc. IEEE Int. Conf. on Communications (ICC), May 2003, vol. 3, pp. 2192 -2196.

[18] J. Zhang, R. A. Kennedy, and T. D. Abhayapala, "Performance of RAKE reception for Ultra Wideband signals in a lognormal fading channel," in Proc. Int. Workship on Ultra Wideband Systems (IWUWBS), June 2003.

[19] A. H. Stroud and D. Secrest, Gaussian Quadrature Formulas, PrenticeHall, Inc., Englewood Cliffs, N.J., 1966. 